\title{
iNEXT-Discovery and Instruct-ERIC: Integrating High-End Services for Translational Research in Structural Biology
}

\author{
Hans Wienk ${ }^{1}$, Lucia Banci ${ }^{2}$, Susan Daenke ${ }^{3}$, Eva Pereiro ${ }^{4}$, Harald Schwalbe ${ }^{5}$, David lan Stuart ${ }^{6}$, Manfred S. \\ Weiss $^{7}$, Anastassis Perrakis ${ }^{1}$ \\ ${ }^{1}$ Division of Biochemistry and Oncode Institute, The Netherlands Cancer Institute, Amsterdam ${ }^{2}$ CERM/CIRMMP, University of Florence ${ }^{3}$ Instruct-ERIC, \\ Oxford University ${ }^{4}$ MISTRAL beamline, Alba Light Source ${ }^{5}$ Center for Biomolecular Magnetic Resonance (BMRZ), Goethe University Frankfurt ${ }^{6}$ Division \\ of Structural Biology, Oxford University ${ }^{7}$ Macromolecular Crystallographyy, Helmholtz-Zentrum Berlin
}

\section{Corresponding Authors}

Hans Wienk

h.wienk@nki.nl

Anastassis Perrakis

a.perrakis@nki.nl

\section{Date Published}

November 16, 2021

\section{Editorial}

The field of structural biology has developed into a powerful system of understanding biology through the atomic details of biomolecular structures, enriching our understanding of biochemical pathways and the molecular basis of diseases, and offering an indispensable tool for translational research.

Over the years, structural biology has gone far beyond providing static structures, to report also on molecular interactions, both in solution and in cells, examining time scales and dynamics. Information can be obtained to explain processes on different size scales, namely, from atoms and individual molecules to entire virus particles and cells. Different technologies provide a synergistic set of experimental approaches, including scattering methods based on X-rays, neutrons, or electrons, e.g., X-ray crystallography (MX) or small-angle X-ray scattering (SAXS); solid state and solution nuclear magnetic resonance (NMR); and single particle reconstruction by cryo-electron microscopy (cryo-EM). Methods such as cryo-EM tomography, cryo-soft X-ray tomography, and in-

\section{Citation}

Wienk, H.,Banci, L.,Daenke, S.,Pereiro, E.,Schwalbe, H.,Stuart, D.I.,Weiss, M.S.,Perrakis, A. iNEX Discovery and Instruct-ERIC: Integrating High-End Services for Translational Research in Structural Biology. J. Vis. Exp. (177), e63435, doi:10.3791/63435 (2021).

DOI

$10.3791 / 63435$
URL

jove.com/video/63435 cell NMR add to this arsenal of approaches to study macromolecular systems, especially in connection with other imaging tools. Together with additional biophysical methods that provide complementary information on affinities and time scales of molecular interaction, integrative structural biology nowadays offers a formidable package for understanding life and developing drugs, biologics (antibodies, nanobodies, vaccines), biomaterials and other biological agents, such as enzymes that can be used to degrade plastic and help the environment.

Early on, the European Commission (EC) recognized the benefits that structural biology research has for society, and since the 1980s Europe strongly invested in opening up expensive structural biology equipment free-of-charge to the larger scientific community. This democratization of access to multi-million Euro facilities was a driving force and a cornerstone for developing the area of structural biology internationally. This placed Europe at the cutting edge of the field, thereby enabling a strong industrial sector and contributing to local economies. 
The EC cherishes a long and impressive tradition of external researcher's access and training in structural biology technologies. Following the successful iNEXT program (Infrastructure for NMR, EM and X-rays for Translational research) that allowed free-of-charge structural biology access to high-end facilities and all European researchers until the end of 2019, the Horizon2020 project iNEXTDiscovery started in February 2020 for another fouryear period of fully funded access to structural biology infrastructures. In parallel, Instruct-ERIC is a Landmark of the European Strategy Forum on Research Infrastructures, constituting a sustainable stronghold in structural biology for the European and international scientific communities. Together, they aspire to engage life scientists and others in structural biology research, and for that they offer access to some of the best structural biology expertise, technologies and facilities worldwide, to researchers from academia and industry alike. Together with additional partners, they also organize workshops and hands-on training.

Because of the Covid19-pandemic, traveling to iNEXTDiscovery and Instruct-ERIC research facilities has been largely impaired, essentially prohibiting handson training opportunities. To partially compensate for this, iNEXT-Discovery and Instruct-ERIC both arranged webinar series (https://inext-discovery.eu/events/what-caninext-discovery-do-for-us, https://instruct-eric.eu/content/ instructeric-webinar-series-structure-meets-function), as well as this special Methods-issue of the Journal of Visualized Experiments. Here, you will find detailed descriptions not only for several of our mainstream access procedures, but also for specialized routines that are under development in the field of integrative structural biology. While our methods are described in this issue in detail, some require expertise and equipment that is hard to establish locally. The Instruct-
ERIC and iNEXT-Discovery facilities are available to provide these methods, including the production of the biomolecules needed for structural studies, with the help and supervision of highly skilled local staff.

\section{Improved methods for determining macromolecular}

\section{structures}

For the last decade, cryo-EM developed into a highly popular technology for determining macromolecular complex structures. The team from Leeds University (UK) provides an overview of all the steps involved in single-particle EM analysis, focusing on variables that can be optimized during the workflow and troubleshooting of common issues ${ }^{1}$. With online microscope operation by external users becoming standard, protocols for efficient remote operation and imaging are required. With single-particle analysis, vitrified samples are being imaged to produce movies showing particles in random orientations. An image processing workflow identifies the parameters for reconstructing the specimen under study. The software Scipion from CSIC (ES) provides all tools to create this workflow, also allowing straightforward traceability of the results ${ }^{2}$.

Collection of X-ray diffraction data on very small crystalline samples offers great research opportunities as well as technical challenges. The Diamond (UK) team presents the use of cryo-EM tools to prepare hundreds of microcrystals on a single grid with minimal surrounding liquid on a low-noise support, before collecting data in a dedicated beamline environment that includes a vacuum in the $X$ ray path before and after the sample ${ }^{3}$. Serial X-ray data collection in synchrotrons is also a key for studying other difficult crystalline systems. In another contribution, Diamond shows step-by-step instructions, figures, and videos for data 
collection using a variety of crystal-delivery systems, through a series of examples ${ }^{4}$.

\section{Modern integrative structural biology in drug discovery}

X-ray crystallography is well-established for fragment-based drug-discovery and is already commonly used to identify hits that can be smoothly developed onto lead candidates. Teams from Diamond ${ }^{5}$, Helmholtz-Zentrum Berlin (DE) and MAX IV $(\mathrm{SE})^{6}$, and the EMBL Grenoble unit $(\mathrm{FR})^{7}$ describe their latest developments in automating workflows for efficient target crystallization, fragment soaking, crystal mounting, crystallography-driven fragment screening, and structure deposition. Their pipelines are fairly automated and being used by external researchers worldwide. They have been applied to already more than 100 targets (often remotely) to identify hits for further compound design and structureassisted lead development.

NMR is also commonly used for fragment screening in drug development campaigns, targeting either proteins or polynucleotides, either soluble or membrane bound. The Goethe University in Frankfurt (DE) presents optimized NMR screening pipelines, reducing manual intervention during experimentation and analyses ${ }^{8}$. Their sample changers handle hundreds of NMR samples in a single run during several days of uninterrupted experimentation and automated analysis.

In addition, biophysical methods are complementary to X-ray and NMR. The team from the Netherlands Cancer Institute $(\mathrm{NL})$ presents a workflow using nano-differential scanning calorimetry for thermal shift assays to measure changes in target melting temperature upon addition of fragments ${ }^{9}$. Prerequisites regarding protein quality and concentration are followed by an analytical protocol for experimental design, data collection, and analysis.

\section{Towards cellular structural biology}

Cryo-electron tomography is often the method of choice for looking into macromolecular complexes in cells. Images from vitrified samples are acquired at different tilt angles, and a 3D-tomogram reconstructed, from which sub-volumes are extracted to give higher resolution by sub-tomogram averaging. As electrons interact strongly with matter, such studies require specimens of less than $200 \mathrm{~nm}$ thickness. The CEITEC team (CZ) presents a protocol ${ }^{10}$ for preparing cellular lamellae after plunge freezing. Even though automation in cryo-ET has made impressive progress, hours of data collection are required before tilt series acquisition takes place, and targets are typically still identified by visual grid inspection. An automation protocol was prepared by EMBL Heidelberg (DE) to map grids and grid squares, to select targets and to set up tilt series acquisition ${ }^{11}$.

The ALBA (ES) and Diamond synchrotrons together discuss the pitfalls and advantages of an alternative to cryo-EM tomography that allows examining intact cells, namely, cryo-soft X-ray tomography (cryo-SXT) ${ }^{12}$. Adapting superresolution techniques to cryogenic temperatures allows correlative imaging with cryo-ET and cryo-SXT. Also, highthroughput cryo-structured illumination microscopy (cryoSIM) doubles the resolution of conventional fluorescence, leading to the specific location of molecules or structures in thick samples. This can be correlated with the cryo-SXT to pinpoint specific features within the cellular context ${ }^{13}$.

The University of Utrecht (NL) team reports sample preparation and high-sensitivity solid-state NMR experiments that can be used to study challenging physiological systems, including natural membranes and protein-lipid- 
glycan systems ${ }^{14}$. In addition, in-cell solution NMR is rapidly evolving to elucidate functional processes. The CERM/ CIRMMP (IT) team describes how to assemble and operate bioreactors that allows replenishment of nutrients and oxygen for living cells inside the NMR probe at the core of a high-field magnet ${ }^{15}$. This allows extended measurements and time-resolved experiments, for example, for monitoring intracellular protein-ligand interactions.

\section{Concluding remarks}

This JoVE issue is fully dedicated to recent structural biology developments and applications in European research infrastructures. Together, iNEXT-Discovery and InstructERIC allow structural biology specialists, but increasingly also non-specialist life scientists and other researchers, to pursue their research aims by offering state-of-the-art services.

\section{Disclosures}

The authors have nothing to disclose.

\section{Acknowledgments}

This JoVE Methods Collection issue is financed by iNEXTDiscovery, project number 871037 , funded by the Horizon 2020 program of the European Commission.

\section{References}

1. White, J.B.R. et al. Single particle cryo-electron microscopy: From sample to structure. Journal of Visualized Experiments: JoVE. (171), e62415 (2021).

2. Jiménez-Moreno, A. et al. Cryo-EM and single-particle analysis with scipion. Journal of. Visualized Experiments: JoVE. (171), e62261 (2021).
3. Crawshaw, A. et al. A sample preparation pipeline for microcrystals at the VMXmbeamline. Journal of Visualized Experiments: JoVE. (172), e62306 (2021).

4. Horrell, S. et al. Fixed target serial data collection at diamond light source. Journal of Visualized Experiments: JoVE. (168), e62491 (2021).

5. Douangamath, A. et al. Achieving efficient fragment screening at XChemfacility at diamond light source. Journal of Visualized Experiments: JoVE. (171), e62414 (2021).

6. Wollenhaupt, J. et al. Workflow and tools for crystallographic fragment screening at the helmholtzzentrum Berlin. Journal of Visualized Experiments: JoVE. (169), e62208 (2021).

7. Cornaciu, I. et al. The automated crystallography pipelines at the EMBL HTX facility in grenoble. Journal of Visualized Experiments: JoVE. (172), e62200 (2021).

8. Berg, $H$. et al. NMR-based fragment screening in a minimum sample but maximum automation mode. Journal of Visualized Experiments: JoVE. (172), e62262 (2021).

9. Ahmad, M.U.D. et al. Nano-differential scanning fluorimetry for screening in fragment-based lead discovery. Journal of Visualized Experiments: JoVE. (171), e62469 (2021).

10. Moravcová, J., Pinkas, M., Holbová, R., Nováček, J. Preparation and CryoFIB micromachining of Saccharomyces cerevisiae for CryoElectrontomography. Journal of Visualized Experiments: JoVE. e62351 (2021).

11. Weis, F., Hagen, W. J. H., Schorb, M., Mattei, S. Strategies for optimization of cryogenic electron 
tomography data acquisition. Journal of Visualized

Experiments: JoVE. (169), e62383 (2021).

12. Groen, J. et al. A 3D cartographic description of the cell by cryosoft X-ray tomography. Journal of Visualized Experiments: JoVE. (169), e62190 (2021).

13. Vyas, N. et al. Cryo-structured illumination microscopic data collection from cryogenically preserved cells. Journal of Visualized Experiments: JoVE. (171), e62274 (2021).

14. Beriashvili, D., Schellevis, R. D., Napoli, F., Weingarth, M., Baldus, M. High-resolution studies of proteins in natural membranes by solid-state NMR. Journal of Visualized Experiments: JoVE. (169), e62197 (2021).

15. Barbieri, L., Luchinat, E. Monitoring protein-ligand interactions in human cells by real-time quantitative incell NMR using a high cell density bioreactor. Journal of Visualized Experiments: JoVE. (169), e62323 (2021). 